\title{
Management challenges in tuberculosis and HIV
}

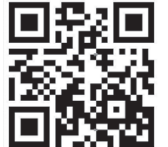

Globally, South Africa (SA) is disproportionately affected by the epidemics of tuberculosis (TB) and the human immunodeficiency virus-1 (HIV-1). The intersection of these two diseases has resulted in an unprecedented disease burden. It is estimated that $12.2 \%$ of South Africans are HIV-infected - a total of 6.4 million people, the largest number in any country in the world. ${ }^{[1]}$ SA has the second highest annual incidence of TB after Swaziland approximately $1 \%$ of the population develop active TB disease each year (an estimated 530000 people in 2012). ${ }^{[2]}$ While SA comprises $0.7 \%$ of the world's population, it is estimated that of all cases of HIVassociated TB that occur worldwide annually, $30 \%$ are in SA. ${ }^{[2]}$

Important gains have been made in the public health response to HIV in the past decade. Starting in 2004, the public sector antiretroviral therapy (ART) programme has enabled over 2 million South Africans to start ART - the largest ART treatment programme in the world. ${ }^{[1]}$ Modelling studies have suggested that ART, when initiated before advanced immunosuppression, allows for a nearnormal life expectancy. ${ }^{[3]}$ An expanded treatment programme has reversed the alarming decreases in life expectancy of South Africans in the 1990s. In rural KwaZulu-Natal, life expectancy rose by 11.3 years between 2003 and 2011. ${ }^{[4]}$ The prevention of mother-tochild transmission (PMTCT) scale-up of antiretroviral strategies, starting with single-dose nevirapine, then adding zidovudine and most recently offering all pregnant mothers triple-drug ART, has dramatically reduced the transmission rates and consequently the prevalence of HIV in the paediatric population. However, HIV incidence rates remain high in the adolescent and adult population owing to sexual transmission. ${ }^{[1]}$

Despite the abovementioned gains, TB and HIV, often as co-infection, remain the most important causes of morbidity and mortality among adult South Africans. ${ }^{[5]}$ HIV-related complications are the most common cause for admission to hospital medical wards in SA. At Khayelitsha Hospital in Cape Town, $>50 \%$ of patients admitted to the medical wards are HIV-infected; many of them also have active TB ( $G$ Meintjes - unpublished data). Therefore, while SA has the largest ART programme in the world, there has not been the same dramatic impact in the prevention of HIV opportunistic infections as in industrialised countries when ART was first introduced. ${ }^{[6]}$ There are many reasons for this, including the following: the number of people living with HIV infection in SA; many patients are diagnosed with HIV when they have advanced immunosuppression; some patients experience virological failure while on ART owing to adherence problems; some patients disengage from care and default ART; even with successful immune reconstitution on ART, HIV-infected patients remain at higher risk of TB disease than HIV-uninfected people in the same community; and the high rates of transmission of TB infection in many communities.

In light of this TB and HIV disease burden, this edition of CME focuses on a number of key topics in the management of HIV, TB and co-infection. Six topics were chosen because these are common clinical scenarios in which diagnosis and management can be challenging and for which there are recent research findings to inform changes in clinical practice.

Wasserman and Meintjes ${ }^{[7]}$ review HIV-associated TB, focusing on diagnostic advances, complications that arise when providing simultaneous treatment for TB and HIV, when to start ART in HIVinfected TB patients, and use of isoniazid preventive therapy (IPT) for TB. The additive benefit of IPT in patients on ART in preventing incident $\mathrm{TB}$ has recently been shown. ${ }^{[8]}$

About 15000 cases of multidrug-resistant TB were diagnosed in SA in 2012. ${ }^{[2]}$ This condition is difficult to manage, particularly as current treatments are less effective than first-line TB treatment, of prolonged duration (18 - 24 months), and difficult to tolerate - with significant toxicities. Outcomes are currently particularly poor for patients with extensively drug-resistant TB (DR-TB). ${ }^{[9]}$ Hughes and Osman, ${ }^{[10]}$ both of whom have expertise in managing DR-TB at primary care level, provide practical insights on its diagnosis and management and also cover new drug options that are becoming available.

$\mathrm{TB}$ meningitis has the highest mortality rate of all forms of TB - up to $60 \%$ in patients with HIV co-infection. ${ }^{[1]}$ Patients who survive, frequently remain with a neurological disability. Diagnosis is made challenging by the limited sensitivity of TB diagnostic tests in the cerebrospinal fluid. The clinical course may be complicated by the immune reconstitution inflammatory syndrome in HIV-infected patients who start ART. An approach to these issues, informed by recent research findings, is covered in the article by Marais and Wilkinson. ${ }^{[12]}$

After TB, cryptococcal meningitis is probably the most important co-infection contributing to mortality in HIV-infected adults in SA. More than 6000 cases were diagnosed in laboratories in SA in 2013. ${ }^{[13]}$ Management of this condition involves initial amphotericin B-based combination antifungal therapy and subsequent consolidation and maintenance with fluconazole, control of raised intracranial pressure with therapeutic lumbar punctures and initiation of ART $4-6$ weeks after diagnosis. ${ }^{[14]}$ Govender and Dlamini ${ }^{[15]}$ discuss issues related to cryptococcal meninigitis prevention and management.

Finally, there are two articles that focus on paediatric HIV management. Adherence to ART is a critical determinant of its long-term success. Adherence is particularly challenging in adolescents. Fairlie et al. ${ }^{[16]}$ address the medical aspects of ART management in adolescents, as well as approaches to optimise adherence and ensure successful transition to adult care when appropriate. While new infections in children have decreased owing to SA's PMTCT programme, vertical transmission of

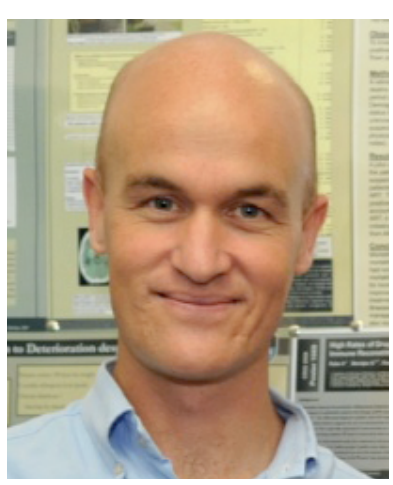
HIV still occurs. Frigati et al. ${ }^{[17]}$ deal with ART management of paediatric patients prior to adolescence, emphasising the importance of early diagnosis and early ART initiation to limit morbidity and mortality. This may also potentially limit the size of the HIV viral reservoir.

Graeme Meintjes

Guest editor

graemein@mweb.co.za 